\title{
Association between the Delta Estimated Glomerular Filtration Rate and the Prevalence of Monoclonal Gammopathy of Undetermined Significance in Korean Males
}

\author{
Tae-Dong Jeong, Woochang Lee, Sail Chun, and Won-Ki Min \\ Department of Laboratory Medicine, Asan Medical Center, University of Ulsan College of Medicine, 88 Olympic-ro 43-gil, \\ Songpa-gu, Seoul 138-736, Republic of Korea \\ Correspondence should be addressed to Won-Ki Min; wkmin@amc.seoul.kr
}

Received 24 February 2014; Revised 12 April 2014; Accepted 22 April 2014; Published 8 May 2014

Academic Editor: Patrizia Cardelli

Copyright (C) 2014 Tae-Dong Jeong et al. This is an open access article distributed under the Creative Commons Attribution License, which permits unrestricted use, distribution, and reproduction in any medium, provided the original work is properly cited.

\begin{abstract}
Background. We investigated the association between the reduction in the estimated glomerular filtration rate (eGFR) and the prevalence of monoclonal gammopathy of undetermined significance (MGUS) in Korean males. Methods. We enrolled 723 healthy Korean males. Serum creatinine concentration, serum electrophoresis, serum immunofixation, and the serum free light chain assay were performed. We calculated delta eGFR per year $(\Delta \mathrm{eGFR} / \mathrm{yr})$. The prevalence of MGUS was compared based on the $\Delta \mathrm{eGFR} / \mathrm{yr}$ and age group. Results. Thirteen (1.8\%) of 723 participants exhibited the monoclonal band on serum immunofixation. Prevalence of MGUS by age group was $0.00 \%$ ( $0 / 172$ for 40 years), $1.63 \%$ (6/367 for 60 years), and $3.80 \%$ (7/184 for $>60$ years). The median decrease in $\triangle \mathrm{eGFR} / \mathrm{yr}$ was $5.3 \%$. The prevalence of MGUS in participants in their $50 \mathrm{~s}$ with $>5.3 \%$ decline in $\Delta \mathrm{eGFR} / \mathrm{yr}$ was significantly higher than those with $<5.3 \%$ decrease in $\Delta \mathrm{eGFR} / \mathrm{yr}(3.16 \%$ versus $0.00 \% ; P=0.049)$. The prevalence of MGUS in participants in their $50 \mathrm{~s}$ with $>5.3 \%$ decrease in $\Delta \mathrm{eGFR} / \mathrm{yr}$ was similar to that of healthy males in their $60 \mathrm{~s}$. Conclusion. Using the rate of reduction in $\triangle \mathrm{eGFR} / \mathrm{yr}$ in healthy Korean males who had their serum creatinine level checked regularly may increase the MGUS detection rate in clinical practice.
\end{abstract}

\section{Introduction}

Monoclonal gammopathy of undetermined significance (MGUS) is the most common plasma cell disorder, and its prevalence increases with age [1-4]. The prevalence of MGUS is also affected by race and sex, but MGUS generally develops in older people, African-Americans, and males with a slight predominance [1-5]. In previous studies, the prevalence of MGUS was $4.9 \%$ in Caucasian males $>60$ years old [2] and $3.8 \%$ in Korean males $>65$ years old [3].

Several studies of the prevalence of MGUS stratified by age category in each race have been conducted. However, to the best of our knowledge, none has reported the correlation between the degree of estimated glomerular filtration rate (eGFR) reduction and the prevalence of MGUS. And the association between measurement of free kappa and lambda light chains by the serum FLC assay and the prevalence of MGUS has not been well documented either. Thus, in this study we investigated the correlation between the degree of egfr reduction and MGUS prevalence among Korean healthy males.

\section{Materials and Methods}

2.1. Subjects. The study included Korean males over 40 years old who visited the hospital for regular health checkups between June 2011 and March 2012. A total of 723 males had regular checkups during the period. According to the review of the electronic medical records, all of the study subjects have not been diagnosed with MGUS previously. This study was approved by the Institutional Review Board of ASAN Medical Center (approval no. 2012-0065).

2.2. Specimen and Data Collection. After general blood chemistry, the remaining $1 \mathrm{~mL}$ of serum was collected in two microtubes and stored at $-70^{\circ} \mathrm{C}$. One of the two microtubes 
TABLE 1: Baseline demographic chracteristics of the study population stratified by age.

\begin{tabular}{|c|c|c|c|c|}
\hline Variable & $40-49$ yrs & $50-59$ yrs & $\geq 60$ yrs & All \\
\hline$N$ & 172 & 367 & 184 & 723 \\
\hline Age (yrs) & $47.0 \pm 1.7$ & $54.3 \pm 2.9$ & $65.4 \pm 5.2$ & $55.4 \pm 7.4$ \\
\hline Interval of health checkup (days) & $395.6 \pm 97.3$ & $394.5 \pm 91.7$ & $420.2 \pm 113.4$ & $401.3 \pm 99.4$ \\
\hline Current sCr (mg/dL) & $0.989 \pm 0.123$ & $0.996 \pm 0.155$ & $1.015 \pm 0.155$ & $0.999 \pm 0.148$ \\
\hline Previous sCr (mg/dL) & $0.943 \pm 0.113$ & $0.941 \pm 0.117$ & $0.968 \pm 0.129$ & $0.949 \pm 0.120$ \\
\hline Current eGFR $\left(\mathrm{mL} / \mathrm{min} / 1.73 \mathrm{~m}^{2}\right)$ & $82.6 \pm 11.5$ & $80.2 \pm 12.6$ & $75.6 \pm 12.8$ & $79.6 \pm 12.7$ \\
\hline Previous GFR (mL/min/1.73 m²) & $87.7 \pm 12.6$ & $85.3 \pm 11.9$ & $79.8 \pm 12.7$ & $84.5 \pm 12.6$ \\
\hline$\Delta \mathrm{eGFR} / \mathrm{yr}(\%)$ & $-5.1 \pm 9.0$ & $-5.6 \pm 8.5$ & $-4.7 \pm 8.0$ & $-5.3 \pm 8.5$ \\
\hline$\kappa \mathrm{FLC}(\mathrm{mg} / \mathrm{L})$ & $9.77(2.38-25.91)$ & $10.76(2.17-292.53)$ & $11.54(2.17-1,265.95)$ & $11.10(2.17-1,265.95)$ \\
\hline$\lambda$ FLC $(\mathrm{mg} / \mathrm{L})$ & $11.13(6.38-30.31)$ & $11.71(2.51-34.71)$ & $12.06(2.51-34.71)$ & $11.84(2.51-34.71)$ \\
\hline$\kappa / \lambda$ ratio & $0.84(0.19-1.99)$ & $0.91(0.33-44.26)$ & $0.94(0.33-156.68)$ & $0.92(0.19-156.68)$ \\
\hline
\end{tabular}

Data expressed as means \pm standard deviation or medians (range).

Abbreviations: CKD-EPI: Chronic Kidney Disease Epidemiology Collaboration; eGFR: estimated glomerular filtration rate; FLC: free light chain; MDRD; Modification of Diet in Renal Disease; sCr: serum creatinine.

was used for serum protein electrophoresis (sPEP) and serum immunofixation electrophoresis (sIFE), while the other was used for the serum free light chain (sFLC) assay. Electronic medical records were used to collect participant data, such as age, date of current health checkup, date of previous health checkup, and current and previous serum creatinine levels.

2.3. Laboratory Tests. Serum creatinine levels of the participants during the study period were measured using a Toshiba 200-FR Neo (Toshiba Medical Systems Co., Tokyo, Japan) instrument with the IDMS-traceable calibrator (c.f.a.s calibrator, Roche Diagnostics, Indianapolis, IN, USA). Previous serum creatinine levels were measured using the Toshiba 200-FR Neo with the same IDMS-traceable calibrator. The Hydrasys 2 (Sebia, Evry, France) instrument was used to perform both sPEP and sIFE based on the manufacturer's instructions. The sIFE was performed to detect kappa and lambda free light chains in sera. The sFLC assay was analyzed using SPAplus (Binding Site, Birmingham, UK) instrument with Freelite (Binding Site) reagents. Both kappa and lambda free light chain concentrations were measured quantitatively to calculate the kappa/lambda FLC ratio. The reference range for the sFLC ratio was $0.26-1.65$ [6].

2.4. Calculating eGFR and $\triangle e G F R$. All participants were Korean males. Their current and previous eGFR values were calculated using the four-variable MDRD study equation $\left(\mathrm{eGFR}=175 \times\right.$ standardized serum creatinine $^{-1.154} \times$ age $\left.{ }^{-0.203}\right)$. The delta eGFR $(\triangle \mathrm{eGFR}, \%)$ was calculated as the difference between current eGFR and previous eGFR $[100 \times$ (current eGFR-previous eGFR)/previous eGFR], whereas the annual rate of decline in eGFR $[\Delta \mathrm{eGFR} / \mathrm{yr}(\%)]$ was determined as the difference between the follow-up and baseline eGFR values, with this value divided by the time interval [100 $\times($ current eGFR-previous eGFR)/previous eGFR $] \times$ (365/ $\Delta$ days).

2.5. Definition of MGUS. MGUS is defined as serum monoclonal protein $<3 \mathrm{~g} / \mathrm{dL}$, clonal bone marrow clonal plasma cells $<10 \%$, and absence of end organ damage such as hypercalcemia, renal insufficiency, anemia, and bone lesions (CRAB) that can be attributed to the plasma cell proliferation disorder [7]. In this study,MGUS was defined as an absence of CRAB symptoms and presence of monoclonal protein confirmed by sIFE. The CRAB symptoms were identified through review of the electronic medical records including laboratory findings.

2.6. Statistical Analyses. The participants were grouped by age $(40,50$, and $>60$ years) and $\Delta \mathrm{eGFR} / \mathrm{yr}$ (classified into two groups by median $\Delta \mathrm{eGFR} / \mathrm{yr}$ ). The prevalence of MGUS was calculated for each group and the differences were analyzed by the chi-square test or Fisher's exact test. SPSS version 19.0 (SPSS, Inc., Chicago, IL, USA) was used for statistical analyses. A $P$ value $<0.05$ was considered to indicate significance.

\section{Results}

3.1. Baseline Characteristics. The mean age of the participants was $55.4 \pm 7.4$ years (range, $41-90$ years) and the average health checkup interval was $401.3 \pm 99.4$ days (range, 202768 days). The current serum creatinine level increased by an average of $0.051 \pm 0.089 \mathrm{mg} / \mathrm{dL}$ from the previous serum creatinine level. eGFR decreased by an annual average of $5.3 \pm$ $8.5 \%$, and 363 participants had $\geq 5.3 \%$ reduction. Median values of serum kappa FLC concentration, serum lambda FLC concentration, and the serum kappa/lambda FLC ratio were $11.10 \mathrm{mg} / \mathrm{L}, 11.84 \mathrm{mg} / \mathrm{L}$, and 0.92 , respectively. The baseline demographic chracteristics of the study population stratified by age are summarized in Table 1.

3.2. sPEP, sIFE, and sFLC Assays. The monoclonal protein was detected in 9 participants by sPEP, 13 by sIFE, and 21 by the sFLC ratio, respectively. Four participants had the monoclonal protein in all three tests. The monoclonal protein was detected in 4 participants by only sIFE and in 17 by only the sFLC assay. 


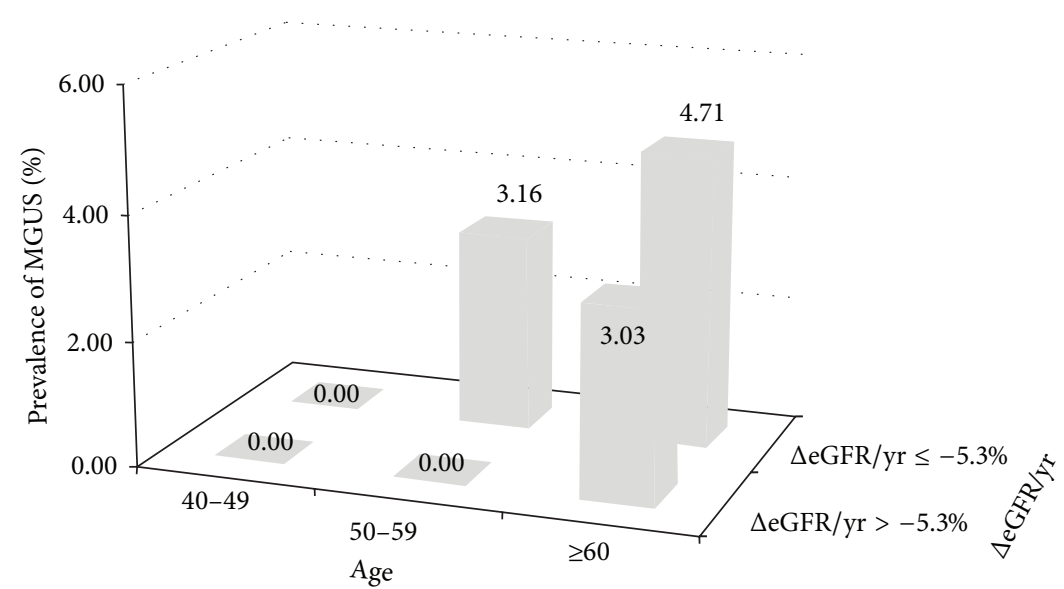

Figure 1: Prevalence of monoclonal gammopathy of undetermined significance in Korean males based on delta estimated glomerular filtration rate and age.

3.3. Prevalence of MGUS. Thirteen (1.80\%) of the seven hundred and twenty-three participants had the monoclonal band on sIFE. The prevalence of MGUS by age group was $0.00 \%$ (0/172, 40 years of age), $1.63 \%$ (6/367, 50 years of age), and $3.80 \%(7 / 184, \geq 60$ years of age) (Table 2$)$.

The MGUS prevalence in the group with $\geq 5.3 \%$ decline in $\Delta \mathrm{eGFR} / \mathrm{yr}$ was $2.75 \%(10 / 363)$, almost threefold that in the other groups $(0.83 \%, 3 / 360)$; however, the difference was not significant $(P=0.089)$. In an age-stratified analyses, the prevalence of MGUS in 50s with $\geq 5.3 \%$ decline in $\Delta \mathrm{eGFR} / \mathrm{yr}$ group was significantly higher than those with $<5.3 \%$ decline in $\Delta \mathrm{eGFR} / \mathrm{yr}$ group $(3.16 \%$ versus $0.00 \% ; P=0.049)$ (Figure 1). In the 60 years of age or older group, however, the prevalence of MGUS showed no significant difference based on $\Delta \mathrm{eGFR} / \mathrm{yr}(4.71 \%$ versus $3.03 \% ; P=0.438$ ) (Figure 1 ).

The MGUS prevalence in participants with kappa or lambda FLC concentrations higher than the reference value on sFLC was $10.91 \%(6 / 55)$, significantly higher than the $1.05 \%$ (7/668) in those with a normal serum kappa and lambda FLC concentration.

\section{Discussion}

We investigated the association between $\Delta \mathrm{eGFR} / \mathrm{yr}$ and prevalence of MGUS in healthy Korean males aged over 40 years. Our results showed that MGUS prevalence values in Korean men in their $40(0.00 \%), 50(1.63 \%)$, and $>60$ years of age $(3.80 \%)$ were similar to MGUS values for Japanese males in their $40(1.2 \%), 50(2.7 \%)$, and $>60$ years of age $(4.3 \%)$ and Chinese men 50-65 years (1.2\%) [8, 9]. To date, the MGUS prevalence in Koreans has been determined only in elderly people $>65$ years [3]. In the present study, the prevalence of MGUS in participants $>60$ years was $3.80 \%$ which was similar to those obtained from elderly Korean male aged over 65 years (3.8\%) [3].

By definition of MGUS, there is no renal insufficiency. However, excess serum free light chains and intact immunoglobulins that exceed the metabolic ability of kidney function in patients with MGUS can accumulate in the kidney, resulting in subclinical renal insufficiency such as a decline in GFR $[10,11]$. On the basis of above consideration, we had assumed that the prevalence of MGUS may be higher in patients with markedly reduction of GFR. And the GFR decline may occur within reference range of serum creatinine. In our study, although the prevalence of MGUS in all participants was not significantly different based on the $\triangle \mathrm{eGFR}$, a statistically significant difference was found in the group in their 50s. The difference between the 50 s group and those $>60$ can be partially explained that MGUS is not always a premalignant lesion. Our study indicated that there was apparent association between the MGUS and $\triangle \mathrm{eGFR}$; however, a large-scale cohort study is needed to reach the statistical significance.

According to the current MGUS management guideline, MGUS does not require aggressive treatment [12]. However, MGUS is an obvious premalignant lesion that requires careful observation because it progresses to multiple myeloma or other plasma cell-related disorders in some patients [12-15]. In this study, the MGUS prevalence in the group with $\geq 5.3 \%$ decline in $\triangle \mathrm{eGFR} / \mathrm{yr}$ was almost threefold that in the other groups. The prevalence of MGUS in participants in their 50s with a high rate of decrease in $\triangle \mathrm{eGFR} / \mathrm{yr}$ was $3.16 \%$, which was similar to that in the group in their 60 s. As $10-20 \%$ of those with MGUS in this group are likely to develop multiple myeloma or diverse malignant plasma cell disorders when they reach 60-70 years of age, early detection of MGUS in 50 s should be considered.

Measurement of serum creatinine level is included in the National Health Screening Program organized by the National Health Insurance Service for Koreans who are required to undergo a regular health checkup every year or 2 years. And the $\triangle \mathrm{eGFR}$ can be calculated from those data. We thought that addition of screening for monoclonal protein in those whose $\Delta \mathrm{eGFR} / \mathrm{yr}$ shows a marked reduction may increase the MGUS diagnostic rate.

In this study, we observed 9 samples of false-negative results for sFLC assay based on the sIFE findings. We thought that possible sources of false-negative results of sFLC 
TABLE 2: Prevalence of monoclonal gammopathy of undetermined significance (MGUS) in Korean males.

\begin{tabular}{lccc}
\hline Age (yrs) & Total number of patients & Number of patients with MGUS & Prevalence of MGUS \\
\hline $40-49$ & 172 & 0 & $0.00 \%$ \\
$50-59$ & 367 & 6 & $1.63 \%$ \\
$\geq 60$ & 184 & 7 & $3.80 \%$ \\
\hline All & 723 & 13 & $1.80 \%$ \\
\hline
\end{tabular}

assay were antigen excess [16], FLC polymerization [17], and polyclonal FLC elevation [18]. On the other hand, sFLC assay demonstrated false-positive results for 17 samples based on the sIFE findings. Seventeen participants were normal on the sIFE and sPEP tests but had an abnormal sFLC ratio. Among them, 15 participants showed kappa clonality which means that their sFLC ratio showed more than 1.65 and the remaining 2 participants demonstrated lambda clonality which means that their sFLC ratio is less than 0.26 (data not shown). It has been well documented that sFLC assay could detect monoclonal protein more sensitive than sPEP and sIFE assay $[6,19]$. So it is not clear whether 17 samples are true false-positive results. These 17 participants may have monoclonal protein in their sera. So we thought that they need to be monitored to identify monoclonal protein.

In conclusion, the prevalence of MGUS in healthy Korean males in their 50 s with a decrease in $\Delta \mathrm{eGFR} / \mathrm{yr}$ of $\geq 5.3 \%$ was significantly higher than those with a decrease in $\Delta \mathrm{eGFR} / \mathrm{yr}$ of $<5.3 \%$. Additionally, the prevalence of MGUS was similar to that of healthy Korean males in their 60 s. Using $\Delta \mathrm{eGFR} / \mathrm{yr}$ in healthy Korean males whose serum creatinine level is checked regularly may facilitate early detection of MGUS.

\section{Conflict of Interests}

The authors have no conflict of interests to declare.

\section{Acknowledgments}

This study was supported by a grant from the Foundation for Industry Cooperation, University of Ulsan funded by DOW Biomedica, Seoul, Korea. The funders had no role in study design, data collection, data analysis, or preparation of the paper.

\section{References}

[1] A. Dispenzieri, J. A. Katzmann, R. A. Kyle et al., "Prevalence and risk of progression of light-chain monoclonal gammopathy of undetermined significance: a retrospective population-based cohort study," The Lancet, vol. 375, no. 9727, pp. 1721-1728, 2010.

[2] R. A. Kyle, T. M. Therneau, S. V. Rajkumar et al., "Prevalence of monoclonal gammopathy of undetermined significance," The New England Journal of Medicine, vol. 354, no. 13, pp. 1362-1369, 2006.

[3] H.-K. Park, K.-R. Lee, Y.-J. Kim et al., "Prevalence of monoclonal gammopathy of undetermined significance in an elderly urban Korean population," American Journal of Hematology, vol. 86, no. 9, pp. 752-755, 2011.
[4] R. K. Wadhera and S. V. Rajkumar, "Prevalence of monoclonal gammopathy of undetermined significance: a systematic review," Mayo Clinic Proceedings, vol. 85, no. 10, pp. 933-942, 2010.

[5] P. Stratta, L. Gravellone, T. Cena et al., "Renal outcome and monoclonal immunoglobulin deposition disease in 289 old patients with blood cell dyscrasias: a single center experience," Critical Reviews in Oncology/Hematology, vol. 79, no. 1, pp. 3142, 2011.

[6] J. A. Katzmann, R. J. Clark, R. S. Abraham et al., "Serum reference intervals and diagnostic ranges for free $\kappa$ and free $\lambda$ immunoglobulin light chains: relative sensitivity for detection of monoclonal light chains," Clinical Chemistry, vol. 48, no. 9, pp. 1437-1444, 2002.

[7] N. Korde, S. Y. Kristinsson, and O. Landgren, "Monoclonal gammopathy of undetermined significance (MGUS) and smoldering multiple myeloma (SMM): novel biological insights and development of early treatment strategies," Blood, vol. 117, no. 21, pp. 5573-5581, 2011.

[8] M. Iwanaga, M. Tagawa, K. Tsukasaki, S. Kamihira, and M. Tomonaga, "Prevalence of monoclonal gammopathy of undetermined significance: study of 52,802 persons in Nagasaki City, Japan," Mayo Clinic Proceedings, vol. 82, no. 12, pp. 1474-1479, 2007.

[9] S. P. Wu, A. Minter, R. Costello et al., "MGUS prevalence in an ethnically Chinese population in Hong Kong," Blood, vol. 121, no. 12, pp. 2363-2364, 2013.

[10] G. Cohen and W. H. Hörl, "Free immunoglobulin light chains as a risk factor in renal and extrarenal complications," Seminars in Dialysis, vol. 22, no. 4, pp. 369-372, 2009.

[11] A. S. Bargnoux, N. Simon, V. Garrigue et al., "Glomerular filtration rate as a determinant of free light chains in renal transplantation," Clinical Biochemistry, vol. 46, no. 16-17, pp. 1764-1766, 2013.

[12] J. Bladé, "Monoclonal gammopathy of undetermined significance," The New England Journal of Medicine, vol. 355, no. 26, pp. 2765-2770, 2006.

[13] J. P. Bida, R. A. Kyle, T. M. Therneau et al., "Disease associations with monoclonal gammopathy of undetermined significance: a population-based study of 17,398 patients," Mayo Clinic Proceedings, vol. 84, no. 8, pp. 685-693, 2009.

[14] R. A. Kyle and S. Kumar, "The significance of monoclonal gammopathy of undetermined significance," Haematologica, vol. 94, no. 12, pp. 1641-1644, 2009.

[15] O. Landgren, G. Gridley, I. Turesson et al., "Risk of monoclonal gammopathy of undetermined significance (MGUS) and subsequent multiple myeloma among African American and white veterans in the United States," Blood, vol. 107, no. 3, pp. 904-906, 2006.

[16] S. S. Levinson, "Hook effect with lambda free light chain in serum free light chain assay," Clinica Chimica Acta, vol. 411, no. 21-22, pp. 1834-1836, 2010. 
[17] C. Li, H. Geng, Z. Yang, and R. Zhong, "Influence of immunoglobulin light chain dimers on the results of the quantitative nephelometric assay," Clinical Laboratory, vol. 57, no. 1-2, pp. 5357, 2011.

[18] S. S. Levinson, "Polyclonal free light chain of Ig may interfere with interpretation of monoclonal free light chain $\kappa / \lambda$ ratio," Annals of Clinical and Laboratory Science, vol. 40, no. 4, pp. 348353, 2010.

[19] A. R. Bradwell, H. D. Carr-Smith, G. P. Mead et al., "Highly sensitive, automated immunoassay for immunoglobulin free light chains in serum and urine," Clinical Chemistry, vol. 47, no. 4, pp. 673-680, 2001. 

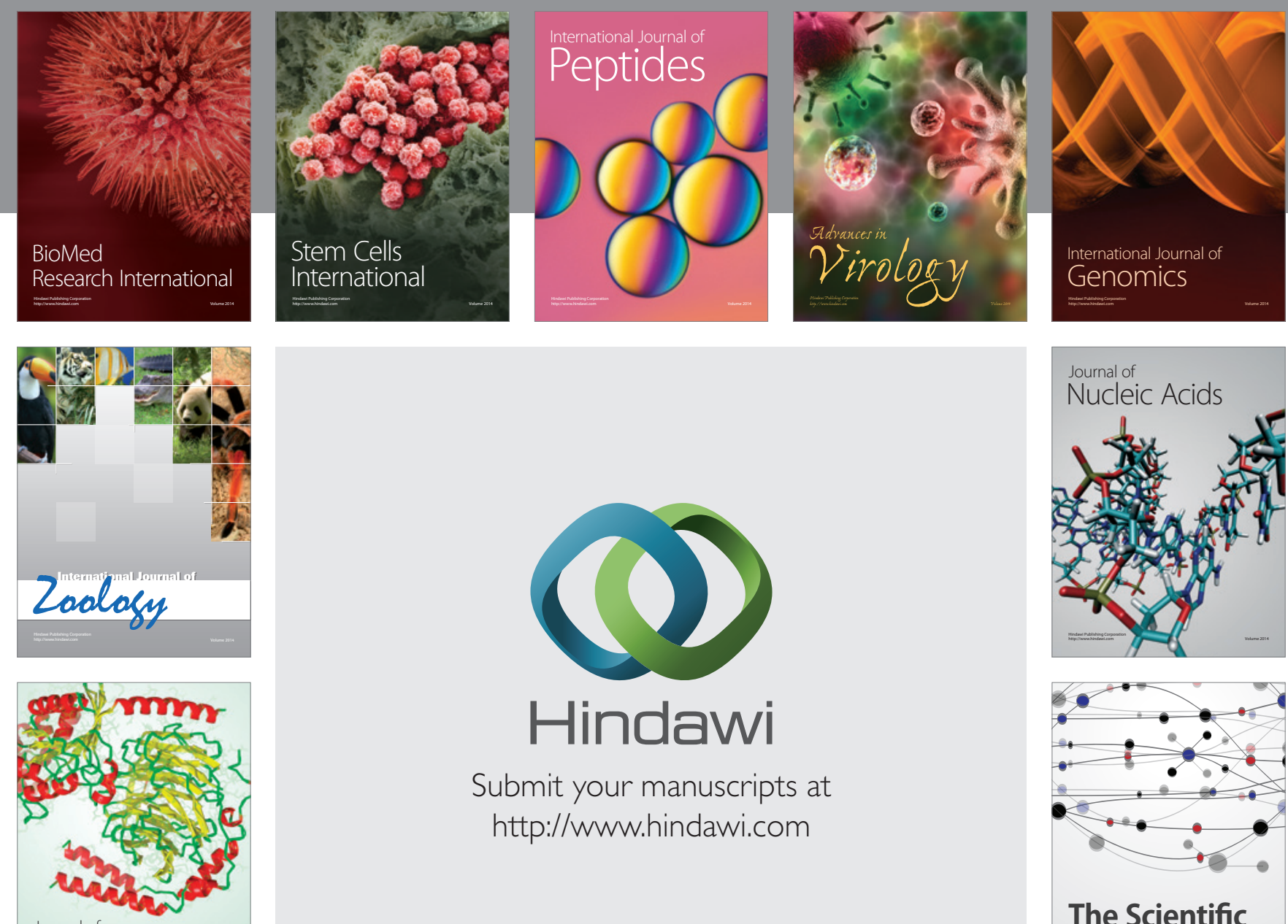

Submit your manuscripts at

http://www.hindawi.com

Journal of
Signal Transduction
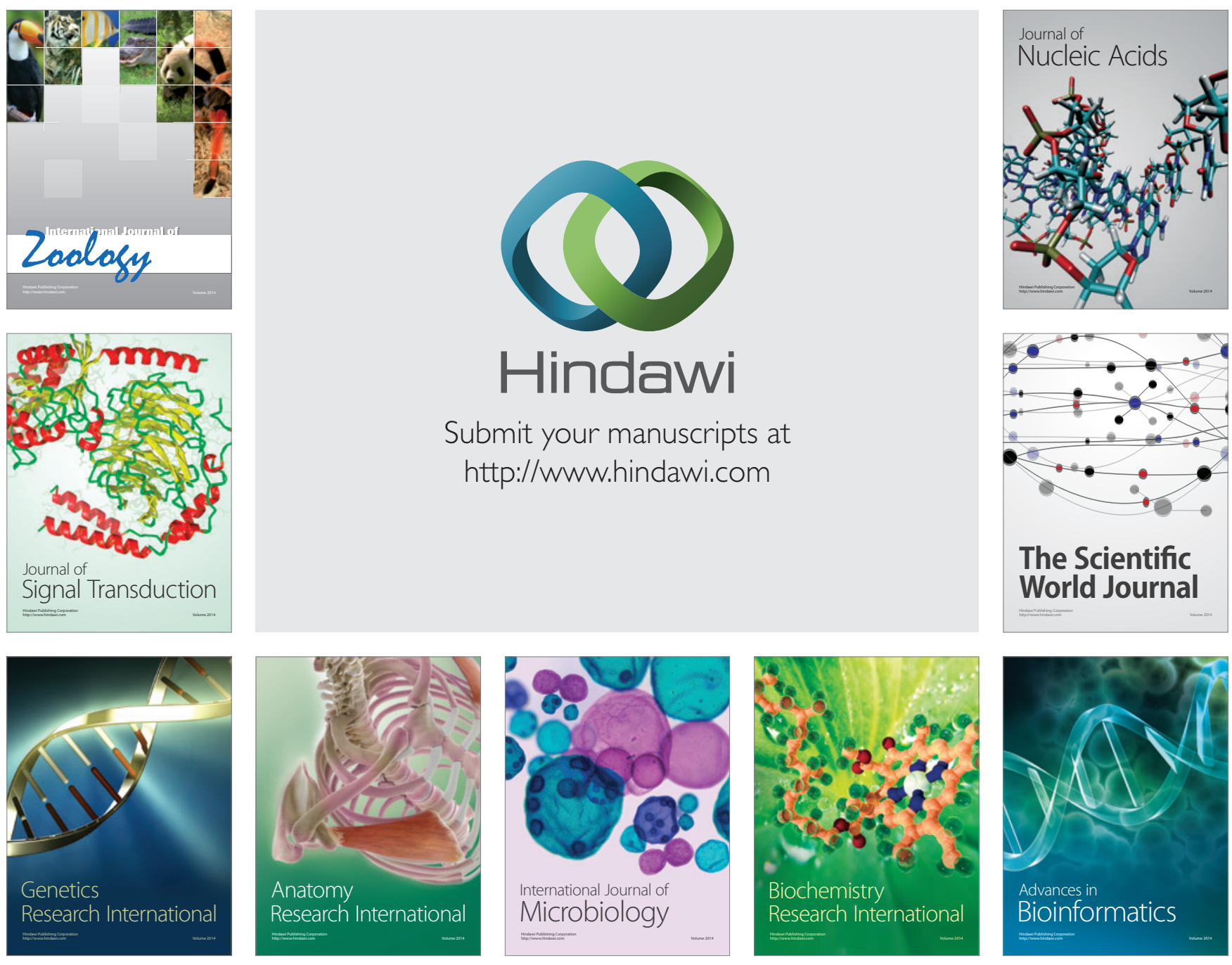

The Scientific World Journal
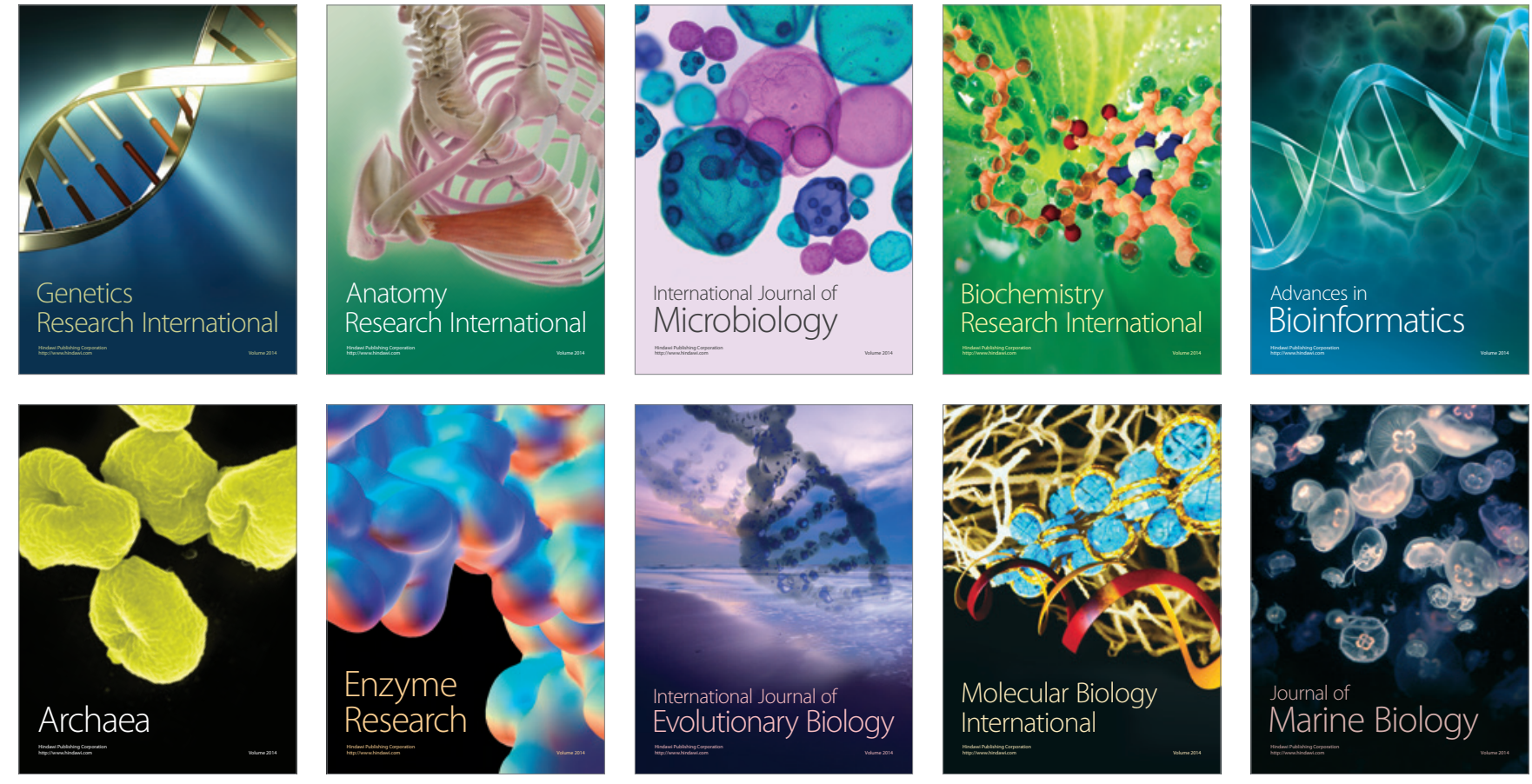